\title{
Attentional demand and memory retrieval in negative priming
}

\author{
Hsuan-Fu Chao $\cdot$ Yei-Yu Yeh
}

Received: 13 October 2005 / Accepted: 4 December 2006 / Published online: 6 January 2007

(C) Springer-Verlag 2007

\begin{abstract}
Negative priming refers to delayed response to previous distractors, and can reflect the operation of attentional selection in prime trials. One important feature of negative priming is that it is modulated by the characteristics of probe trials. The current study manipulated competition from probe distractors and prime-probe similarity to examine the effects of attentional demand and memory retrieval in probe trials. The results demonstrated that the effects of attentional demand and memory retrieval on negative priming were dynamic. Distractor competition in probe trials affected negative priming in Experiment 1, and primeprobe similarity affected negative priming in Experiment 2. Moreover, negative priming in Experiment 3 was observed either when competition from probe distractors was strong or when identical spatial layouts were used in prime-probe couplets. Taken together, either competition from probe distractors or primeprobe similarity of spatial layouts was critical to the manifestation of negative priming at one time. Implications for distractor inhibition and memory retrieval in negative priming were discussed.
\end{abstract}

Y.-Y. Yeh $(\bowtie)$

Department of Psychology, National Taiwan University,

No. 1, Sec. 4, Roosevelt Rd., Taipei 106, Taiwan

e-mail: yyy@ntu.edu.tw

\section{H.-F. Chao ( $ه)$}

Department of Psychology,

Chung Yuan Christian University, No. 200,

Chung Pei Rd, Chung Li 32023, Taiwan

e-mail: hfchao@cycu.edu.tw

\section{Introduction}

Distractors irrelevant to behavioral goals are omnipresent. They influence performance in searching, identifying, and responding to targets. In the search context, the number of distractors increases reaction time (RT) when perception of a target requires feature integration (Treisman \& Gelade, 1980). Target-distractor similarity can also lengthen search time (Duncan \& Humphreys, 1989) to safeguard the accuracy of response choice. Irrelevant distractors can also be beneficial in the search process. Distractors demarcate the spatial layout of a visual scene by their locations around a target to influence visual search through memory retrieval (Chun \& Jiang, 1998, 1999). When the same spatial layouts repeated across blocks of trials, search performance was more efficient for targets occurring in consistent locations than for targets in new configurations.

In a nonsearch context in which a target always occurs in the center of a screen and participants are instructed to ignore flanking distractors, the distractors can also influence performance through their compatibility with the target's response selection (Eriksen \& Eriksen, 1974). Incompatibility delays RT. The effectiveness of selective attention depends upon the degree to which the information processing system can reduce the distractors' influence.

An item, however, might not always serve as a distractor. A target can become a distractor, and a distractor can become a target. When a recently ignored distractor turns into a target, a phenomenon, the negative priming effect, is usually observed (Tipper, 1985; Tipper \& Cranston, 1985). As the name implies, negative priming is the phenomenon of a slower response to 
recently ignored distractors (Fox, 1995; May, Kane, \& Hasher, 1995; Neill, Valdes, \& Terry, 1995; for reviews). This effect is usually evaluated in laboratories by using couplets consisting of a prime trial and a subsequent probe trial. Participants are asked to respond to the target and ignore the distractor in both the prime and probe trials. Negative priming is assessed by comparing the RT to control couplets in which the items in the probe trials and prime trials are unrelated to the RT to ignored repetition (IR) couplets in which the probe target is the prime distractor.

Since Dalrymple-Alford \& Budayr's (1966) first observation of negative priming with a Stroop colornaming task (Stroop, 1935), negative priming has been observed with a variety of stimuli and tasks, including naming (Neill, 1977; Tipper \& Cranston, 1985), location judgment (Tipper, Brehaut, \& Driver, 1990; Tipper, Weaver, \& Houghton, 1994), lexical decision (Yee, 1991), categorization (Chiappe \& MacLeod, 1995; Tipper \& Driver, 1988), perceptual matching (DeSchepper \& Treisman, 1996), and size judgment (MacDonald \& Joordens, 2000; MacDonald, Joordens, \& Seergobin, 1999). In addition, the negative priming effect generalizes across various forms of materials, including simple symbols (Tipper et al., 1990), letters (Tipper \& Cranston, 1985), objects (Tipper, 1985; Tipper \& Driver, 1988), faces (Grison, Tipper, \& Hewitt, 2005), words (Malley \& Strayer, 1995), colored words (Neill, 1977), and nonsense shapes (DeSchepper \& Treisman, 1996).

The phenomenon of negative priming highlights the cost as a result of selective attention that the information processing system must endure after reducing interference from distractors. More importantly, the negative priming effect shows that unattended distractors in many experimental contexts are processed to a semantic level rather than at the level of primitive features (Tipper \& Driver, 1988), indicating where the locus of selective attention can be. Thus, the paradigm of negative priming has become an important methodology that researchers adopt to understand the operation and the cost of selective attention.

However, the effect of negative priming reflects not only attentional selection in prime trials, but also attentional and/or retrieval processes in probe trials. If the effect of negative priming could be totally attributed to distractor inhibition in prime trials (Tipper, 1985; Tipper \& Cranston, 1985), the characteristics of probe trials should not be critical to negative priming. In contrast to this prediction, the characteristics of probe trials can influence the manifestation of negative priming in several ways. Studies of prime-probe similarity indicated that the effect of negative priming was stron- ger when probe trials were similar to their prime trials (e.g., Fox \& de Fockert, 1998; Neill, 1997; Neill \& Valdes, 1996; Neill, Valdes, Terry, \& Gorfein, 1992; but see Lavie \& Fox, 2000; Paquet, 2001; Wong, 2000; Yang, Yeh, \& Chao, 2004). Moreover, several studies revealed that the presence of probe distractors was important to the manifestation of the negative priming effect (Lowe, 1979; Milliken, Joordens, Merikle, \& Seiffert, 1998; Moore, 1994; Tipper \& Cranston, 1985). Negative priming failed to occur when there is no competing distractor in probe trials (Lowe, 1979; Milliken et al., 1998; Moore, 1994; Tipper \& Cranston, 1985; but see Neill, Terry, \& Valdes, 1994; Yee, 1991 for different results; and see Frings \& Wentura, 2006 for a further discussion). And the negative priming effect was reduced when competition from probe distractors was low (Yeh \& Chao, 2004).

The importance of prime-probe similarity in negative priming supported Neill and colleagues' memory retrieval account of negative priming (Neill \& Mathis, 1998; Neill \& Valdes, 1992; Neill et al., 1992). Considering the effect of probe distractors in negative priming, Tipper and Cranston (1985) highlighted the necessity of maintaining a selection state with the presence of a probe distractor for the operation of the inhibitory mechanism, and Houghton and Tipper (1994) stressed the importance of distractor competition in probe trials in the manifestation of the inhibitory effect. It is certain that the effect of prime-probe similarity and probe distractors in negative priming have important implications for theories of negative priming. However, these two factors were examined independently. There is no study investigating the effect of prime-probe similarity and competition from probe distractors in the same experiment.

Examining the effect of prime-probe similarity and competition from probe distractors together is important to the theories of negative priming. It is critical to determine whether prime-probe similarity and competition from probe distractors influence negative priming independently or interactively. It is possible that when competition from probe distractors is strong, higher prime-probe similarity can further increase the magnitude of the negative priming effect because of the selection difficulty caused by strong competition from probe distractors (e.g., Neill \& Mathis, 1998). It is also possible that when strong competition from probe distractors affects the magnitude of negative priming, prime-probe similarity has no effect on negative priming, and vice versa. It would be discussed how theories of negative priming incorporate the effect of primeprobe similarity and competition from probe distractor in the "General discussion". 


\section{Experiment 1}

To manipulate prime-probe similarity without affecting competition from probe distractors, we varied the relative location of the distractors such that the configuration of a flanker-type display (Eriksen \& Eriksen, 1974) was either horizontal or vertical (see Fig. 1 for examples). The same layout was used in each primeprobe couplet for the identical condition. The prime and probe trials displayed different layouts, alternating between vertical and horizontal layouts in the different condition.

To manipulate competition from probe distractors without altering prime-probe similarity of spatial configurations, competition from probe distractors was controlled by using repeated versus nonrepeated stimuli as probe distractors. According to the activation model of Malley and Strayer (1995); see also Strayer and Grison (1999), when a stimulus is repeatedly used in an experiment, it should have higher activation and competes with target processing to a greater extent than a nonrepeated stimulus does. As a result, the negative priming effect is easier to be observed with repeated rather than nonrepeated stimuli (Chao \& Yeh, 2005; Kramer \& Strayer, 2001; Malley \& Strayer, 1995; Strayer \& Grions, 1999). Therefore, it should be more difficult to select a target when its distractor was a well practiced, highly familiar stimulus.

\section{Method}

\section{Participants}

Twenty undergraduate students at National Taiwan University participated in this experiment for a bonus credit in an introductory psychology course. All participants in this and subsequent experiments were naïve to the purpose of the experiment and had normal or corrected-to-normal vision.

\section{Design and stimuli}

This experiment followed a factorial design with three within-subject variables. The three factors were trial

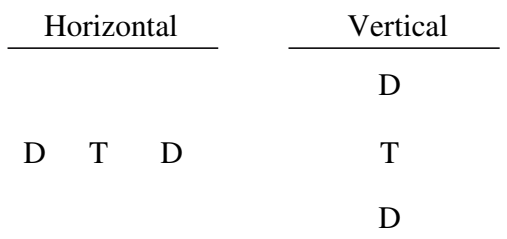

Fig. 1 Spatial configurations used in Experiment 1. $T$ target, $D$ distractor type (IR/control), competition from probe distractors (strong/weak), and prime-probe similarity (identical/ different). There were 30 prime-probe couplets in each of the eight conditions, producing 240 prime-probe couplets in the experiment.

Using the word count database provided by the Ministry of Education Committee for the Promotion and Propagation of the National Language (1997), 165 Chinese character words of high frequency (ranging from 252 to 504 per million) were selected. The number of strokes in these 165 words ranged from 5 to 10 . We divided them into 11 sets of 15 words with similar average word frequency and average number of strokes. Two sets were randomly selected as practice stimuli, and the other nine were used as experimental stimuli.

Four forms of stimuli were constructed before the experiment so that the results could not be the consequence of using a particular type of stimulus assignment. Each form was used with equal frequency across participants. For each form, one set was selected from the nine sets of experimental stimuli as the repeated set. The 15 words in this repeated set were used as prime targets, prime distractors, or probe targets across the entire experiment. These words were also used as probe distractors in the strong competition condition. These 15 words were assigned into each prime-probe couplet with three constraints. First, each word was selected as a prime target, prime distractor, or probe target with equal frequency. Second, each word was assigned into the eight experimental conditions (i.e., trial type $x$ competition from probe distractors $\times$ prime-probe similarity) with equal frequency. Third, four different words were used in each control couplet, as the prime target, the prime distractor, the probe target, and the probe distractor, respectively. Three different words were used in each IR couplet, as the prime target, the prime distractor and the probe target, and the probe distractor, respectively. The remaining eight sets of experimental stimuli were used as probe distractors in the weak competition condition. To generate $30 \mathrm{cou}-$ plets in the 4 conditions in which competition from probe distractors was weak (IR/control $\times$ identical/ different), two sets were used in each cell. The order of presenting couplets was randomized.

A flanker-type display (Eriksen \& Eriksen, 1974) was used. The target was presented in the center of a screen with another word on both the left and right sides. Participants viewed the stimuli at a distance of approximately $60 \mathrm{~cm}$. At the viewing distance, each word subtended a visual angle of $0.67^{\circ}$ vertically and $0.67^{\circ}$ horizontally. The center-to-center distance from the target to a distractor was $1.05^{\circ}$ in visual angle. All stimuli were presented in white on a black background. 


\section{Procedure}

The experiment was run by the DMDX software (Forster $\&$ Forster, 2003). Naming latency was measured with a voice key and the experimenter recorded naming accuracy. Each participant was tested individually.

Participants were asked to name the target in the center as quickly and as accurately as possible. They were also told to ignore the two words adjacent to the target because the distractors could interfere with their response. Twelve practice trials were conducted to assure that participants understood the instruction. Two hundred forty prime-probe couplets were then run in a random sequence. Participants rested after every 30 prime-probe couplets.

The time course of a prime-probe couplet was as follows. A cross in the center of the screen and a "ding" sound were simultaneously presented for $300 \mathrm{~ms}$ as a warning signal. A stimulus display was subsequently presented on the screen until the participant spoke the word or until $3 \mathrm{~s}$ had elapsed. The next warning display was presented with a delay of $200 \mathrm{~ms}$ after the participant said the word. The procedure for presenting prime and probe trials was identical to eliminate observable distinction between a prime trial and a probe trial as a couplet.

\section{Results}

A total of $2.75 \%$ of the trials were excluded from data analysis due to recording failures (e.g., the voice did not register or the participant coughed), or due to errors made in responding to the prime display. Thus, both RT and the percentage of error rates reflect the performance in correctly naming probe targets contingent upon correct response to the prior prime target. We used the same method in subsequent experiments to exclude trials.

The average median $\mathrm{RT}$ in each condition is presented in Table 1 and the effect of negative priming is

Table 1 Averages and standard deviations (SD) of median correct reaction times (RTs) (in ms) of the ignored repetition (IR) condition and the control condition as a function of competition from probe distractors/prime-probe similarity in Experiment 1

\begin{tabular}{llllll}
\hline & \multicolumn{2}{l}{ Trial type } & & \\
\cline { 2 - 3 } & IR & & & Control & \\
\cline { 2 - 3 } \cline { 5 - 6 } & $M$ & & & & \\
& & & & SD \\
\hline Strong/identical & 541.0 & 37.5 & & 525.9 & 39.7 \\
Strong/different & 550.8 & 39.5 & & 531.7 & 42.6 \\
Weak/identical & 533.8 & 33.2 & & 526.9 & 43.4 \\
Weak/different & 539.7 & 40.4 & & 531.7 & 39.9 \\
\hline
\end{tabular}

illustrated in Fig. 2. Median RTs were analyzed by a repeated-measure ANOVA with a 2 (trial type) $\times 2$ (competition from probe distractors) $\times 2$ (primeprobe similarity) within-subject design. All three main effects were significant: trial type $[F(1,19)=15.81$, MSE $=382.09, \quad P<0.01]$, competition from probe distractors $[F(1,19)=4.31, \mathrm{MSE}=174.67, P<0.05]$, and prime-probe similarity $[F(1,19)=7.97, \mathrm{MSE}=$ 216.29, $P<0.05]$. These results showed higher RT in the IR condition, in the strong competition condition, and in the condition where the spatial configuration differed in a couplet. Moreover, the interaction between trial type and competition from probe distractors was significant $[F(1,19)=8.54, \mathrm{MSE}=110.21$, $P<0.01]$. Analysis of simple main effect revealed a significant effect of negative priming in both the strong competition $[F(1,38)=23.87, \mathrm{MSE}=246.15, P<0.01]$ and the weak competition condition $[F(1,38)=4.50$, $P<0.05]$. The interaction reflected that the effect of negative priming was larger with strong competition from probe distractors.

The average percentage of error rates in each condition is presented in Table 2. Analysis of error rates by the repeated-measure ANOVA showed a significant main effect of trial type $[F(1,19)=4.40, \mathrm{MSE}=2.02$, $P<0.05]$ and also of competition from probe distractors $[F(1,19)=7.81, \mathrm{MSE}=5.15, P<0.05]$. The main effect of prime-probe similarity was marginally significant $[F(1,19)=4.05, \mathrm{MSE}=2.06, P=0.056]$. These results showed a lower accuracy in the IR condition, in the strong competition condition, and in the condition of different configurations. The interaction between trial type and competition from probe distractors was significant $[F(1,19)=8.82, \quad \mathrm{MSE}=1.90, \quad P<0.01]$. Analysis of simple main effect showed that the effect of trial type occurred only when competition from probe distractors was strong $[F(1,38)=12.77, \mathrm{MSE}=1.96$, $P<0.01]$ but not when it was weak $[F(1,38)=0.31$, $P>0.20]$.

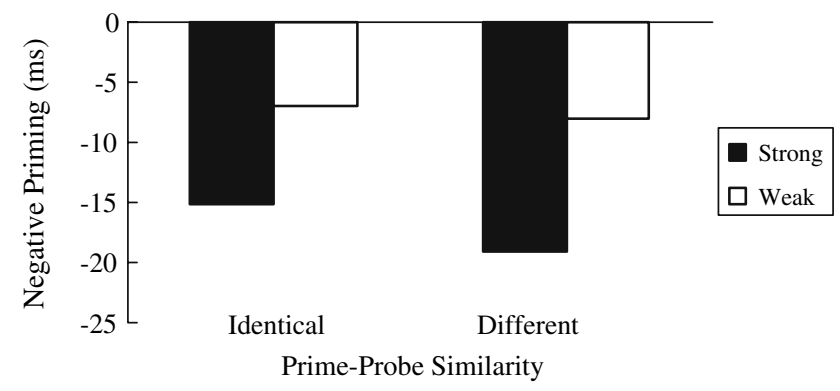

Fig. 2 The effect of negative priming as a function of competition from probe distractors and prime-probe similarity in Experiment 1 
Table 2 Averages and standard deviations (SD) of error rates (in $\%$ ) of the IR condition and the control condition as a function of competition from probe distractors/prime-probe similarity in Experiment 1

\begin{tabular}{llllll}
\hline & \multicolumn{2}{l}{ Trial type } & & \\
\cline { 2 - 3 } & IR & & & Control & \\
\cline { 2 - 5 } & $M$ & SD & & $M$ & SD \\
\hline Strong/identical & 1.6 & 2.8 & 0.3 & 1.0 \\
Strong/different & 2.0 & 2.0 & 1.1 & 2.4 \\
Weak/identical & 0.0 & 0.0 & 0.2 & 0.8 \\
Weak/different & 0.3 & 1.0 & 0.5 & 1.3 \\
\hline
\end{tabular}

\section{Discussion}

Prime-probe similarity did not interact with trial type to influence negative priming although similarity influenced the overall performance. Negative priming was significant regardless of competition from probe distractors but competition imposed by probe distractors modulated the effect. The magnitude of the negative priming effect $(7.5 \mathrm{~ms})$ was smaller in the weak competition condition in which distractors were new contenders in contrast to the strong competition condition $(17.1 \mathrm{~ms})$ in which probe distractors were strong competitors. These findings replicated the effect of the probe distractor competition observed in Yeh and Chao's (2004) study. However, competition from probe distractors had no influence in the control condition ${ }^{1}$. Because negative priming can be observed without the behavioral interference effect (Driver \& Tipper, 1989), we speculated that the negative priming effect may be more sensitive to competition from probe distractors than the interference effect.

The results of Experiment 1 appeared to be against the memory retrieval component in negative priming, showing that competition from probe distractors affected negative priming and prime-probe layout similarity did not. The null finding may have resulted from the use of simple configurations so that the similarity manipulation was not effective. To encourage the use of memory retrieval, the difference between two alternative configurations was increased in Experiment 2, and the variability of alternative configurations was raised in Experiment 3. Thus, the experimental context is more appropriate for testing the effect of primeprobe similarity and the effect of probe distractor competition.

\footnotetext{
${ }^{1}$ We thank Raymond Klein for noting this absence of effect in the control condition.
}

\section{Experiment 2}

We hypothesized that the difference between two configurations used in Experiment 1 was not large enough to heighten the importance of prime-probe similarity. The spatial manipulation was simply a linear transformation with a horizontal array rotating $90^{\circ}$ into a vertical array or vice versa. We thus accentuated the difference by using a linear configuration and a cross configuration in this experiment. The same layout, either a line or a cross, was used in the identical condition for each prime-probe couplet. A line changed into a cross layout or vice versa in the different condition (see Fig. 3).

\section{Method}

\section{Participants}

Twenty-four undergraduate students at National Taiwan University participated in this experiment for a bonus credit in an introductory psychology course.

\section{Design, materials, and procedure}

The experiment followed the same design as Experiment 1 with the same materials and procedure. A cross configuration replaced the vertical configuration.

\section{Results}

We used a cross layout and a horizontal layout to manipulate prime-probe similarity. There are two distractors in the horizontal layout and four in the cross layout. Thus, the effect of distractor competition may differ between these two spatial configurations. Houghton, Tipper, Weaver, and Shore ${ }^{2}$ (1996) manipulated the number of distractors with one or two different stimuli in a prime display. They observed greater interference and reduced negative priming when there were two prime distractors. To uncover the effect of

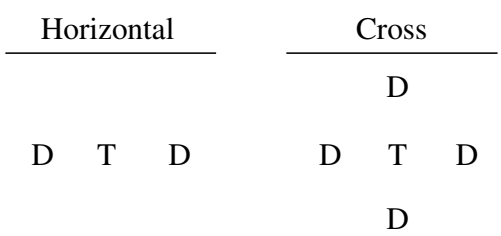

Fig. 3 Spatial configurations used in Experiment 2. $T$ target, $D$ distractor

\footnotetext{
${ }^{2}$ We thank Steven Tipper for suggesting this way of analysis.
} 
distractor number, we analyzed the impact of using different spatial configurations on negative priming.

A total of $2.57 \%$ of the responses were excluded from further analysis. The average median RT in each condition is presented in Table 3, and the effect of negative priming is illustrated in Fig. 4. We analyzed median correct RTs with a 2 (trial type) $\times 2$ (competition from probe distractors) $\times 2$ (spatial layout of prime trial) $\times 2$ (spatial layout of probe trial) repeated measures ANOVA. The main effect of trial type was significant $[F(1,23)=31.87, \mathrm{MSE}=263.79, P<0.001]$, showing negative priming with higher RT in the IR condition than in the control condition. Furthermore, the effect of trial type interacted with the layout of prime trial and the layout of probe trial, resulting in a three-way interaction, $F(1,23)=7.05$, MSE $=241.43$, $P<0.05$.

Negative priming was significant no matter whether both prime and probe trials were identically in the horizontal layout $[F(1,92)=22.13, \quad \mathrm{MSE}=264.08$, $P<0.001]$ or in the cross layout $[F(1,92)=12.09$, $P<0.01]$. When different layouts were used in primeprobe couplets, a different result pattern emerged. Negative priming was not significant when prime trials were in the horizontal layout and probe trials were in the cross layout, $F(1,92)=0.82, P>0.15$. It was significant when prime trials were in the cross layout and probe trials were in the horizontal layout, $F(1,92)$ $=4.82, P<0.05$.

The main effect of competition from probe distractors was also significant, $F(1,23)=5.13, \mathrm{MSE}=344.03$, $P<0.05$, showing higher interference when selection in probe distractors was difficult. There was a two-way interaction between the competition from probe

Table 3 Averages and standard deviations (SD) of median correct RTs (in ms) of the IR condition and the control condition as a function of spatial layout of prime/probe trial and competition from probe distractors in Experiment 2

\begin{tabular}{|c|c|c|c|c|}
\hline & \multicolumn{4}{|c|}{ Trial type } \\
\hline & \multicolumn{2}{|l|}{ IR } & \multicolumn{2}{|c|}{ Control } \\
\hline & $M$ & SD & $M$ & SD \\
\hline \multicolumn{5}{|c|}{ Horizontal-Horizontal } \\
\hline Strong & 536.3 & 51.1 & 521.1 & 50.3 \\
\hline Weak & 535.0 & 53.4 & 518.9 & 56.7 \\
\hline \multicolumn{5}{|c|}{ Cross-Cross } \\
\hline Strong & 535.5 & 55.7 & 521.9 & 50.0 \\
\hline Weak & 530.8 & 58.6 & 521.3 & 53.9 \\
\hline \multicolumn{5}{|c|}{ Horizontal-Cross } \\
\hline Strong & 540.4 & 57.2 & 539.0 & 51.1 \\
\hline Weak & 529.8 & 52.1 & 525.2 & 45.8 \\
\hline \multicolumn{5}{|c|}{ Cross-Horizontal } \\
\hline Strong & 533.4 & 55.1 & 524.8 & 44.0 \\
\hline Weak & 531.6 & 52.9 & 525.6 & 54.8 \\
\hline
\end{tabular}

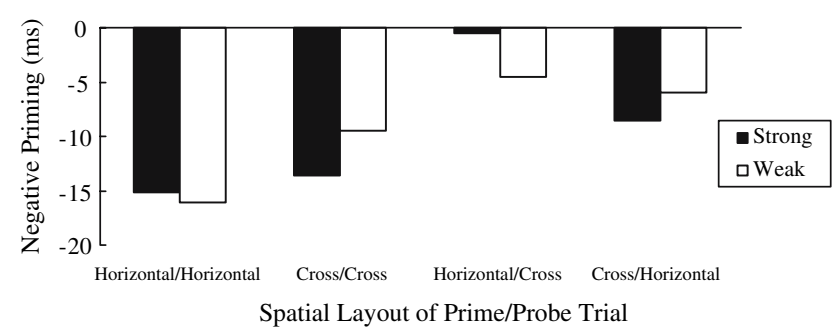

Fig. 4 The effect of negative priming as a function of competition from probe distractors and spatial layout of prime/probe trial in Experiment 2

distractors and the layout of the probe trial, $F(1,23)$ $=6.29, \mathrm{MSE}=150.33, P<0.05$. Analysis of simple main effect revealed that the effect of selection difficulty was significant when a probe was in the cross layout $[F(1,46)=10.71$, MSE $=247.18, P<0.01]$, but not in the horizontal layout $[F(1,46)=0.26, P>0.15]$. Other main effects and interactions were not significant $(P \mathrm{~s}>0.10)$.

The average percentage of error rates in each condition is presented in Table 4. Results showed a significant main effect of competition from probe distractors $[F(1,23)=7.43, \mathrm{MSE}=2.82, P<0.05]$, with a higher error rate when selection was more difficult than the error when selection was easy for both the IR and control conditions. No other effects were significant $(P \mathrm{~s}>0.10)$.

\section{Discussion}

Prime-probe similarity moderates the effect of negative priming. Negative priming was larger when prime and probe trials were in the same spatial configuration

Table 4 Averages and standard deviations (SD) of error rates (in $\%$ ) of the IR condition and the control condition as a function of spatial layout of prime/probe trial and competition from probe distractors in Experiment 2

\begin{tabular}{|c|c|c|c|c|}
\hline & \multicolumn{4}{|c|}{ Trial type } \\
\hline & \multicolumn{2}{|l|}{ IR } & \multicolumn{2}{|c|}{ Control } \\
\hline & $M$ & SD & $M$ & $\mathrm{SD}$ \\
\hline \multicolumn{5}{|c|}{ Horizontal-Horizontal } \\
\hline Strong & 0.6 & 2.0 & 0.3 & 1.5 \\
\hline Weak & 0.3 & 1.5 & 0.0 & 0.0 \\
\hline \multicolumn{5}{|c|}{ Cross-Cross } \\
\hline Strong & 0.6 & 2.0 & 0.3 & 1.7 \\
\hline Weak & 0.0 & 0.0 & 0.3 & 1.4 \\
\hline \multicolumn{5}{|c|}{ Horizontal-Cross } \\
\hline Strong & 1.1 & 3.2 & 0.6 & 1.9 \\
\hline Weak & 0.3 & 1.4 & 0.0 & 0.0 \\
\hline \multicolumn{5}{|c|}{ Cross-Horizontal } \\
\hline Strong & 0.6 & 1.9 & 0.6 & 2.0 \\
\hline Weak & 0.0 & 0.0 & 0.0 & 0.0 \\
\hline
\end{tabular}


$(-13.6 \mathrm{~ms})$ than when prime and probe trials were in different spatial configurations $(-5.2 \mathrm{~ms})$. In comparison to Experiment 1, a larger contrast in spatial configuration led to the impact of prime-probe similarity on negative priming.

The number of prime distractors modulated the negative priming effect, as Houghton et al. (1996) found. More interestingly, the number of prime distractors modulated negative priming only when dissimilar layouts were used in prime-probe couplets. When identical layout was used, the negative priming effect was not significantly different $(P>0.40)$ between the horizontal-horizontal condition $(-15.6 \mathrm{~ms})$ and the crosscross condition $(-11.5 \mathrm{~ms})$. When different layouts were used in couplets, negative priming was absent when a horizontal prime display changed into a cross probe display $(-3.0 \mathrm{~ms})$ and significant when a cross prime display changed into a horizontal probe display $(-7.3 \mathrm{~ms})$. The number of prime/probe distractors mattered only when the prime and probe trials differed in spatial configuration.

In contrast to the results of Experiment 1, level of competition from probe distractors did not influence negative priming. Nevertheless, the overall performance in this experiment indicated that the manipulation of competition from probe distractors was successful. When two distractors entered the race with a probe target in a horizontal layout, competition from probe distractors did not matter. When four distractors entered the race, difficult selection led to a slower response than easy selection. The impact of selection difficulty on performance was the same for both IR and control conditions.

Although competition from probe distractors did not affect negative priming, competition from prime distractors influenced negative priming when memory retrieval was not instigated by dissimilar layouts. Assuming that competition from distractors was stronger with a cross layout to enlarge the inhibitory effect, negative priming was affected by the number of prime distractors when different layouts were used in a prime-probe couplet. That is, negative priming was significant when the prime trial was in the cross layout (four distractors) rather than in the horizontal layout (two distractors). This interaction suggested that when prime-probe similarity was low, negative priming was observed only when competition from prime distractors was strong.

In contrast to Experiment 1, the current findings demonstrated the component of memory retrieval in negative priming. The magnitude of negative priming effect was larger when prime and probe layouts were identical than when the two layouts differed. The effect of competition from distractors was observed only when prime-probe similarity was low (i.e., prime and probe trials were in different configurations). We conducted Experiment 3 to further understand the relationship among prime-probe similarity, competition from probe distractors, and negative priming.

\section{Experiment 3}

In this experiment we raised the likelihood of observing the effect of prime-probe contextual similarity on negative priming by increasing the variety of configurations while keeping the number of distractors constant. Goldstone, Medin, and Halberstadt (1997) demonstrated that context is important in similarity judgments. They suggested that the importance of a particular dimension is dynamically altered as a function of its variability. A dimension becomes important when the magnitude of difference between options is great, when the number of qualitatively distinct values is large, or when variation is introduced.

Eight types of configurations were used (see Fig. 5 for examples) in an imaginary $3 \times 3$ square. The target was always located in the center of the square, which was also at the center of the screen. Two distractors were arranged in two of the eight surrounding locations to form a linear layout with the target in one set, and to form a non-linear right angle composition in another set. We manipulated three levels of primeprobe similarity: identical, similar, and dissimilar to examine the effect of similarity on negative priming.

In the identical condition, the spatial configuration of the probe trial was exactly the same as the spatial configuration of the prime trial. A prime-probe couplet in the similar condition were not identical, but shared the same linear or non-linear layout. The layout configuration changed formation from linear to nonlinear or vice versa in the dissimilar condition, under the constraint that the probe distractors were located

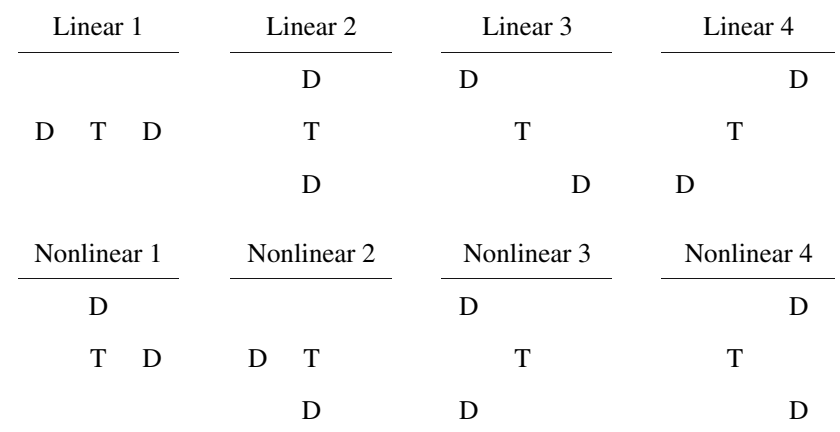

Fig. 5 Spatial configurations used in Experiment 3. $T$ target, $D$ distractor 
at positions different from those of prime distractors such that location information could not exert any influence.

\section{Method}

\section{Participants}

Twenty-four undergraduate students at National Taiwan University participated in this experiment for a bonus credit in an introductory psychology course.

\section{Design and materials}

The experiment followed a 2 (trial type) $\times 2$ (competition from probe distractors) $\times 3$ (prime-probe similarity) within-subject design. There were 24 prime-probe couplets in each condition and 288 prime-probe couplets in the experiment.

One hundred ninety-two Chinese words of high frequency were selected from a word database (the Ministry of Education Committee for the Promotion and Propagation of the National Language, 1997). The word count frequency ranged from 252 to 656 per million, and the number of strokes of each word ranged from 5 to 10 . These 192 words were divided into 8 sets of stimuli, and matched in word frequency and number of strokes. One set was randomly selected as practice materials and the other seven were used for experimental trials. One of the seven sets was randomly chosen as the repeated set in the difficult selection condition for each participant, under the same constraints as in Experiment 1. Materials in the remaining six sets were used as probe distractors in the easy selection conditions.

\section{Procedure}

The procedure followed the one used in Experiment 1 with minor variations. The number of practice trials was 12 , the number of prime-probe couplets was 288 , and there was a pause for rest after every 32 couplets.

\section{Results}

Approximately $3.24 \%$ of the couplets were excluded from further analysis. The average median RTs in each condition are presented in Table 5 and the effect of negative priming is illustrated in Fig. 6. Results from a repeated-measures ANOVA of median correct RTs showed a significant main effect of trial type $[F(1,23)=$ 13.74, $\mathrm{MSE}=228.63, P<0.01]$, competition from probe distractors $[F(1,23)=11.71, \mathrm{MSE}=220.89, P<0.01]$,
Table 5 Averages and standard deviations (SD)of median correct $\mathrm{RTs}$ (in $\mathrm{ms}$ ) of the IR condition and the control condition as a function of competition from probe distractors/prime-probe similarity in Experiment 3

\begin{tabular}{llllll}
\hline & \multicolumn{2}{l}{ Trial type } & & \\
\cline { 2 - 3 } & IR & & & \multicolumn{2}{l}{ Control } \\
\cline { 2 - 3 } \cline { 5 - 6 } & $M$ & & SD & & \\
\cline { 2 - 3 } & & & & SD \\
\hline Strong/identical & 532.5 & 41.4 & & 521.5 & 49.7 \\
Strong/similar & 536.6 & 41.7 & & 528.6 & 44.7 \\
Strong/dissimilar & 538.5 & 44.4 & & 529.0 & 41.5 \\
Weak/identical & 528.4 & 41.9 & & 515.9 & 44.4 \\
Weak/similar & 527.1 & 45.2 & & 528.7 & 47.6 \\
Weak/dissimilar & 525.5 & 45.2 & 525.3 & 47.8 \\
\hline
\end{tabular}

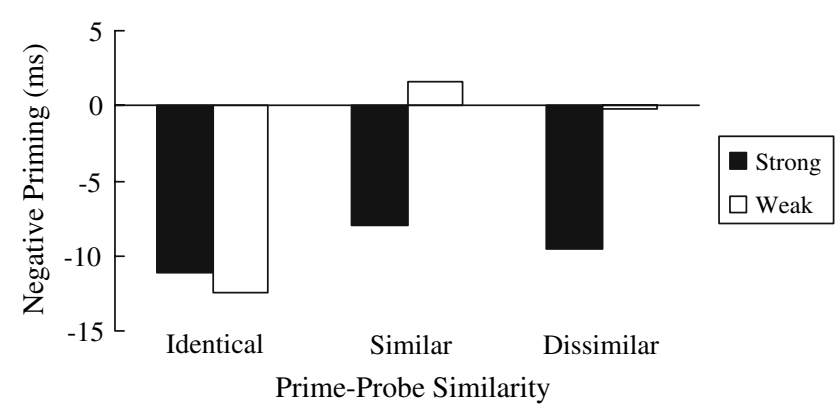

Fig. 6 The effect of negative priming as a function of competition from probe distractors and prime-probe similarity in Experiment 3

and prime-probe similarity $[F(2,46)=4.26, \mathrm{MSE}=$ 216.09, $P<0.05]^{3}$. These main effects reflect a higher $\mathrm{RT}$ in the IR condition than in the control condition, and a higher RT under difficult selection than in the easy selection condition. A REGWQ test at a significance level of 0.05 , of the main effect of prime-probe similarity showed a faster RT in the identical condition than in the other two conditions and RTs were not significantly different between the similar and dissimilar conditions.

The interaction of trial type and prime-probe similarity was significant $[F(2,46)=3.79, \mathrm{MSE}=131.33$, $P<0.05]$. Yet, the three-way interaction that could have uncovered how prime-probe similarity and competition from probe distractors interactively influence negative priming was not significant $(F<1)$. We conducted planned contrasts to examine the negative

\footnotetext{
$\overline{3 \text { To examine }}$ whether different layout configuration interacted with negative priming, we further conducted a four-way repeated measures ANOVA that included layout type as a factor. Although responding to a target in a linear layout was significantly slower than that in the non-linear layout $[F(1,23)=5.25$, $\mathrm{MSE}=488.27, P<0.05]$, this effect did not interact with other variables. Thus, only the effect of competition and prime-probe similarity were discussed.
} 
priming effect in each condition. When competition from probe distractors was strong, negative priming was significant or near significant in the identical $[F(1,138)$ $=7.17, \mathrm{MSE}=205.73, P<0.01]$, similar $[F(1,138)=$ $3.71, P=0.056]$, and the dissimilar condition $[F(1,138)$ $=5.29, P<0.05]$. On the other hand, when competition was weak, negative priming was significant only in the identical condition $[F(1,138)=9.05, P<0.01]$, but not in the similar $[F(1,138)=0.15, P>0.20]$ or dissimilar condition $[F(1,138)=0.00, P>0.20]$. The reversed result pattern in both the similar and dissimilar conditions suggests that the effect of selection difficulty may be observed only when different configurations are used in prime-probe couplets.

The average percentage of error rates in each condition is presented in Table 6. Results of error rates showed a significant main effect of competition from probe distractors $[F(1,23)=14.73, \quad \mathrm{MSE}=3.90$, $P<0.01]$, with a lower accuracy when selection was difficult for both the IR and control conditions. No other effects were significant $(P \mathrm{~s}>0.20)$.

\section{Discussion}

Replicating the results of Experiment 2, prime-probe similarity moderated the effect of negative priming with a smaller effect when the configuration was not identical in couplets $(4.0 \mathrm{~ms})$ in comparison to when the configuration was identical in couplets $(11.8 \mathrm{~ms})$. Although the three-way interaction was not significant, planned contrasts revealed the effect of competition from probe distractors. When competition was strong, negative priming was observed regardless prime-probe similarity. When competition was weak, negative priming could be observed only when the prime and probe trials shared identical spatial configuration.

Table 6 Averages and standard deviations (SD) of error rates (in $\%$ ) of the IR condition and the control condition as a function of competition from probe distractors/prime-probe similarity in Experiment 3

\begin{tabular}{|c|c|c|c|c|}
\hline & \multicolumn{4}{|c|}{ Trial type } \\
\hline & \multicolumn{2}{|l|}{ IR } & \multicolumn{2}{|c|}{ Control } \\
\hline & $M$ & SD & $M$ & SDE \\
\hline Strong/identical & 1.1 & 2.6 & 0.7 & 1.7 \\
\hline Strong/similar & 1.4 & 2.4 & 0.5 & 1.5 \\
\hline Strong/dissimilar & 1.4 & 2.4 & 1.5 & 2.7 \\
\hline Weak/identical & 0.2 & 0.9 & 0.4 & 1.2 \\
\hline Weak/similar & 0.2 & 0.9 & 0.0 & 0.0 \\
\hline Weak/dissimilar & 0.4 & 1.2 & 0.2 & 0.9 \\
\hline
\end{tabular}

\section{General discussion}

The effects of competition from probe distractors and prime-probe similarity, reflecting the impact of attentional demand and memory retrieval on negative priming, are dynamic. The results of Experiment 1 showed the effect of competition from probe distractors on negative priming. Negative priming was observed when competition was strong. After increasing the perceptual difference between alternative configurations, the effect of prime-probe similarity surfaced in Experiment 2. The effect of negative priming was observed primarily when a probe trial was similar to its prime trial in spatial configuration. The results of Experiment 3 revealed both competition from probe distractors and prime-probe similarity can have an impact on negative priming. The effect of negative priming manifested when competition from probe distractors was strong or when probe trials were identical to their prime trials. Taken together, either competition from probe distractors or prime-probe similarity was critical to negative priming at one time. These findings could shed lights on the operations of the inhibitory and retrieval mechanisms that may underlie negative priming.

If negative priming primarily reflects the operation of attentional inhibition (Houghton \& Tipper, 1994; Tipper, 1985; Tipper \& Cranston, 1985), competition from probe distractors can affect the negative priming effect in two ways. First, strong competition from probe distractors, or difficult selection in probe trials, should increase the perceived necessity for maintaining a selection state (Moore, 1994; Tipper \& Cranston, 1985). Because the selection state can encourage the maintenance of the inhibitory effect, negative priming should be observed only in the presence of strong competitors in probe trials. Second, the computational simulation of Houghton and Tipper (1994) suggested that negative priming results from the competition among the probe distractors and probe targets. When competition from probe distractors is strong, it is easier to observe the effect of negative priming. To incorporate the effect of prime-probe similarity, the inhibition account must assume a retrieval mechanism underlying negative priming. Recently, Grison, Tipper and their colleagues (Grison, Kessler, Paul, Jordan, \& Tipper, 2004; Grison et al., 2005; Tipper, 2001) integrated attentional and retrieval mechanisms in one model, which suggested that the inhibitory effect is stored in memory representations. Therefore, the inhibitory effect is manifested when its memory representations are retrieved. It should be noted that, however, the inhibition account with a retrieval mechanism cannot explain why either 
competition from probe distractors or prime-probe similarity was critical to negative priming at one time.

If negative priming primarily reflects memory retrieval of previous episodes (Mayr \& Buchner, 2006; Neill \& Mathis, 1998; Neill \& Valdes, 1992; Neill et al., 1992), it is expected that prime-probe similarity should affect negative priming by modulating probability of memory retrieval. When probe trials are similar to their prime trials, it is easier to retrieve the episodes of their prime trials. Therefore, these episodes of prime trials affect target processing in probe trials. By adding an assumption that strong competition from probe distractors, or selection difficulty in probe trials, encourages memory retrieval (e.g., Neill \& Mathis, 1998), the memory retrieval account is able to explain the importance of competition from probe distractors on negative priming. That is, when it is difficult to respond to the target when there are competing distractors, memory retrieval can further aid processing of targets. Therefore, strong from probe distractors can increase the effect of negative priming. However, with this assumption, the memory retrieval account should expect largest effect of negative priming with strong competition from probe distractors and high prime-probe similarity, which was not observed in the current study. A possible solution is to further assume that both competition from probe distractors and prime-probe similarity are sufficient to encourage memory retrieval, and their influences on memory retrieval are nonadditive. The underlying assumption of this solution is that level of competition from distractors can also serve as a contextual cue. When competition from prime distractors and competition from probe distractors are at the same level, such as the strong competition condition in the current study, memory retrieval is encouraged. Both assumptions are testable and can be examined in future studies.

If both attentional inhibition and memory retrieval can lead to negative priming independently (Chao \& Yeh, 2004; Kane, May, Hasher, Rahhal, \& Stoltzfus, 1997; May et al., 1995), competition from probe distractors matters when negative priming is caused by attentional inhibition and prime-probe similarity matters when negative priming is due to memory retrieval. One further assumption is required to explain the dynamic effects of competition from probe distractors and prime-probe similarity on negative priming. That is, only one process dominates the manifestation of negative priming at one time, and the context determines which process dominates. When the spatial configuration of a target and its distractors can serve as an effective retrieval cue, the effect of memory retrieval dominates. On the other hand, when memory retrieval is not effective and selection difficulty is encountered, the effect of attentional inhibition dominates. Therefore, either competition from probe distractors or prime-probe similarity mattered at one time.

The current study demonstrates that distractor competition in probe trials and prime-probe similarity can determine whether the negative priming effect is present or not. The current study indicates that the phenomena of negative priming cannot be understood without studying the roles of probe trials in it. Specifically, the effects of both attentional demands and memory retrieval in probe trials should be considered.

Acknowledgments This study was supported by a grant from the National Science Council of Taiwan to Y.-Y.Y. (NSC92-2413H-002-025). We thank Bruce Milliken, Steven Tipper, Raymond Klein, and two anonymous reviewers for their critical comments on an earlier version of this draft. We thank the Ministry of Education Committee for the Promotion and Propagation of the National Language for providing the database from which we selected stimuli.

\section{References}

Chao, H.-F., \& Yeh, Y.-Y. (2004). Distractors of low activation can produce negative priming. Memory \& Cognition, 32, 979-989.

Chao, H.-F., \& Yeh, Y.-Y. (2005). Location negative priming in identity discrimination relies on location repetition. Perception \& Psychophysics, 67, 789-801.

Chiappe, D. L., \& MacLeod, C. M. (1995). Negative priming is not task bound: A consistent pattern across naming and categorization tasks. Psychonomic Bulletin \& Review, 2, 364-369.

Chun, M. M., \& Jiang, Y. (1998). Contextual cueing: Implicit learning and memory of visual context guides spatial attention. Cognitive Psychology, 36, 28-71.

Chun, M. M., \& Jiang, Y. (1999). Top-down attentional guidance based on implicit learning of visual covariation. Psychological Science, 10, 360-365.

Dalrymple-Alford, E. C., \& Budayr, D. (1966). Examination of some aspects of the Stroop color-word test. Perceptual \& Motor Skills, 23, 1211-1214.

DeSchepper, B., \& Treisman A. (1996). Visual memory for novel shapes: Implicit coding without attention. Journal of Experimental Psychology: Learning, Memory, and Cognition, 22, 27-47.

Driver, J., \& Tipper, S. P. (1989). On the nonselectivity of "selective" seeing: Contrasts between interference and priming in selective attention. Journal of Experimental Psychology: Human Perception and Performance, 15, 304-314.

Duncan, J., \& Humphreys, G. W. (1989). Visual search and stimulus similarity. Psychological Review, 96, 433-458.

Eriksen, B. A., \& Eriksen, C. W. (1974). Effects of noise letters upon the identification of a target letter in a nonsearch task. Perception \& Psychophysics, 16, 155-160.

Forster, K. I., \& Forster, J. C. (2003). DMDX: A windows display program with millisecond accuracy. Behavior Research Methods, Instruments, \& Computers, 35, 116-124.

Fox, E. (1995). Negative priming from ignored distractors in visual selection: A review. Psychonomic Bulletin \& Review, 2, 145-173. 
Fox, E., \& de Fockert, J. W. (1998). Negative priming depends on prime-probe similarity: Evidence for episodic retrieval. Psychonomic Bulletin \& Review, 5, 107-113.

Frings, C., \& Wentura, D. (2006). Strategy effects counteract distractor inhibition: Negative priming with constantly absent probe distractors. Journal of Experimental Psychology: Human Perception and Performance, 32, 854-864.

Goldstone, R. L., Medin, D. L., \& Halberstadt, J. (1997). Similarity in context. Memory \& Cognition, 25, 237-255.

Grison, S., Kessler, K., Paul, M. A., Jordan, H., \& Tipper, S. P. (2004). Object- and location-based inhibition in goal-directed action: Inhibition of return reveals behavioural, anatomical dissociations and interactions with memory processes. In: G. W. Humphreys, \& J. Riddoch (Eds.), Attention in action. Hove: Psychology Press.

Grison, S., Tipper, S. P., \& Hewitt, O. (2005). Long-term negative priming: Support for retrieval of prior attentional processes. Quarterly Journal of Experiment Psychology, 58A, 11991224.

Houghton, G., \& Tipper, S. P. (1994). A model of inhibitory mechanisms in selective attention. In: D. Dagenbach, \& T. Carr (Eds.), Inhibitory processes in attention, memory, and language (pp. 53-112). San Diego: Academic.

Houghton, G., Tipper, S. P., Weaver, B., \& Shore, D. I. (1996). Inhibition and interference in selective attention: Some tests of a neural network model. Visual Cognition, 3, 119-164.

Kane, M. J., May, C. P., Hasher, L., Rahhal, T., \& Stoltzfus, E. R. (1997). Dual mechanisms of negative priming. Journal of Experimental Psychology: Human Perception and Performance, 23, 632-650.

Kramer, A. F., \& Strayer, D. L. (2001). Influence of stimulus repetition on negative priming. Psychology \& Aging, 16, 580587.

Lavie, N., \& Fox, E. (2000). The role of perceptual load in negative priming. Journal of Experimental Psychology: Human Perception and Performance, 26, 1038-1052.

Lowe, D. G. (1979). Strategies, context and the mechanisms of response inhibition. Memory \& Cognition, 7, 382-389.

MacDonald, P. A., \& Joordens, S. (2000). Investigating a memory-based account of negative priming: Support for selection-feature mismatch. Journal of Experimental Psychology: Human Perception and Performance, 26, 1478-1496.

MacDonald, P. A., Joordens, S., \& Seergobin, K. N. (1999). Negative priming effects that are bigger than a breadbox: Attending to distractors does not eliminate negative priming but rather enhance it. Memory \& Cognition, 27, 197207.

Malley, G. B., \& Strayer, D. L. (1995). Effect of stimulus repetition on positive and negative identity priming. Perception \& Psychophysics, 57, 657-667.

May, C. P., Kane, M. J., \& Hasher, L. (1995). Determinants of negative priming. Psychological Bulletin, 118, 35-54.

Mayr, S., \& Buchner, A. (2006). Evidence for episodic retrieval of inadequate prime responses in auditory negative priming. Journal of Experimental Psychology: Human Perception and Performance, 32, 932-943.

Milliken, B., Joordens, S., Merikle, P. M., \& Seiffert, A. E. (1998). Selective attention: A reevaluation of the implications of negative priming. Psychological Review, 105, 203-229.

Moore, C. M. (1994). Negative priming depends on probe-trial conflict: Where has all the inhibition gone? Perception \& Psychophysics, 56, 133-147.

Neill, W. T. (1977). Inhibitory and facilitatory processes in selective attention. Journal of Experimental Psychology: Human Perception and Performance, 3, 444-450.
Neill, W. T. (1997). Episodic retrieval in negative priming and repetition priming. Journal of Experimental Psychology: Learning, Memory, and Cognition, 23, 1291-1305.

Neill, W. T., \& Mathis, K. M. (1998). Transfer-inappropriate processing: Negative priming and related phenomena. In D. L. Medin (Ed.), The psychology of learning and motivation: Advances in research and theory (Vol. 38, pp. 1-44). San Diego: Academic.

Neill, W. T., Terry, K. M., \& Valdes, L. A. (1994). Negative priming without probe selection. Psychonomic Bulletin \& Review, $1,119-121$.

Neill, W. T., \& Valdes, L. A. (1992). Persistence of negative priming: Steady state or decay? Journal of Experimental Psychology: Learning, Memory, and Cognition, 18, 565-576.

Neill, W. T., \& Valdes, L. A. (1996). Facilitatory and inhibitory aspects of attention. In A. F. Kramer, M. G. H. Coles, \& G. D. Logan (Eds.), Converging operations in the study of visual selective attention (pp. 77-106). Washington, DC: American Psychological Association.

Neill, W. T., Valdes, L. A., \& Terry, K. M. (1995). Selective attention and the inhibitory control of cognition. In F. N. Dempster, \& C. J. Brainerd (Eds.), Interference and inhibition in cognition (pp. 207-261). New York: Academic.

Neill, W. T., Valdes, L. A., Terry, K. M., \& Gorfein, D. S. (1992). Persistence of negative priming: II. Evidence for episodic trace retrieval. Journal of Experimental Psychology: Learning, Memory, and Cognition, 18, 993-1000.

Paquet, L. (2001). Eliminating flanker effects and negative priming in the flankers task: Evidence for early selection. Psychonomic Bulletin \& Review, 8, 301-306.

Strayer, D. L., \& Grison, S. (1999). Negative identity priming is contingent on stimulus repetition. Journal of Experimental Psychology: Human Perception and Performance, 25, 24-38.

Stroop, J. R. (1935). Studies of interference in serial verbal reactions. Journal of Experimental Psychology, 18, 643-662.

The Ministry of Education Committee for the Promotion and Propagation of the National Language. (1997). The frequency statistics of the words and phrases in the compiled materials of the Concise Mandarin Dictionary. Taipei, Taiwan: The Ministry of Education.

Tipper, S. P. (1985). The negative priming effect: Inhibitory priming by ignored objects. Quarterly Journal of Experimental Psychology, 37A, 571-590.

Tipper, S. P. (2001). Does negative priming reflect inhibitory mechanisms? A review and integration of conflicting views. Quarterly Journal of Experimental Psychology, 54A, 321343.

Tipper, S. P., Brehaut, J. C., \& Driver, J. (1990). Selection of moving and static objects for the control of spatially directed action. Journal of Experimental Psychology: Human Perception and Performance, 16, 492-504.

Tipper, S. P., \& Cranston, M. (1985). Selective attention and priming: Inhibitory and facilitatory effects of ignored primes. Quarterly Journal of Experimental Psychology, 37A, 591611.

Tipper, S. P., \& Driver, J. (1988). Negative priming between pictures and words in a selective attention task: Evidence for semantic processing of ignored stimuli. Memory \& Cognition, 16, 64-70.

Tipper, S. P., Weaver, B., \& Houghton, G. (1994). Behavioural goals determine inhibitory mechanisms of selective attention. Quarterly Journal of Experimental Psychology, 47A, 809-840.

Treisman, A. M., \& Gelade, G. (1980). A feature-integration theory of attention. Cognitive Psychology, 12, 97-136. 
Wong, K. F. E. (2000). Dissociative prime-probe contextual similarity effects on negative priming and repetition priming: A challenge to episodic retrieval as a unified account of negative priming. Journal of Experimental Psychology: Learning, Memory, and Cognition, 26, 1411-1422.

Yang, T.-Y., Yeh, Y.-Y., \& Chao, H.-F. (2004). The effect of contextual similarity on negative priming. Chinese Journal of Psychology, 46, 15-27.
Yee, P. L. (1991). Semantic inhibition of ignored words during a figure classification task. Quarterly Journal of Experimental Psychology, 43A, 127-153.

Yeh, Y.-Y., \& Chao, H.-F. (2004). Probe distractors can influence negative priming by perceptual grouping. Perception \& Psychophysics, 66, 208-218. 